\title{
Observatorio de bibliometría y cienciometría USTA FICHA BIBLIOMÉTRICA
}

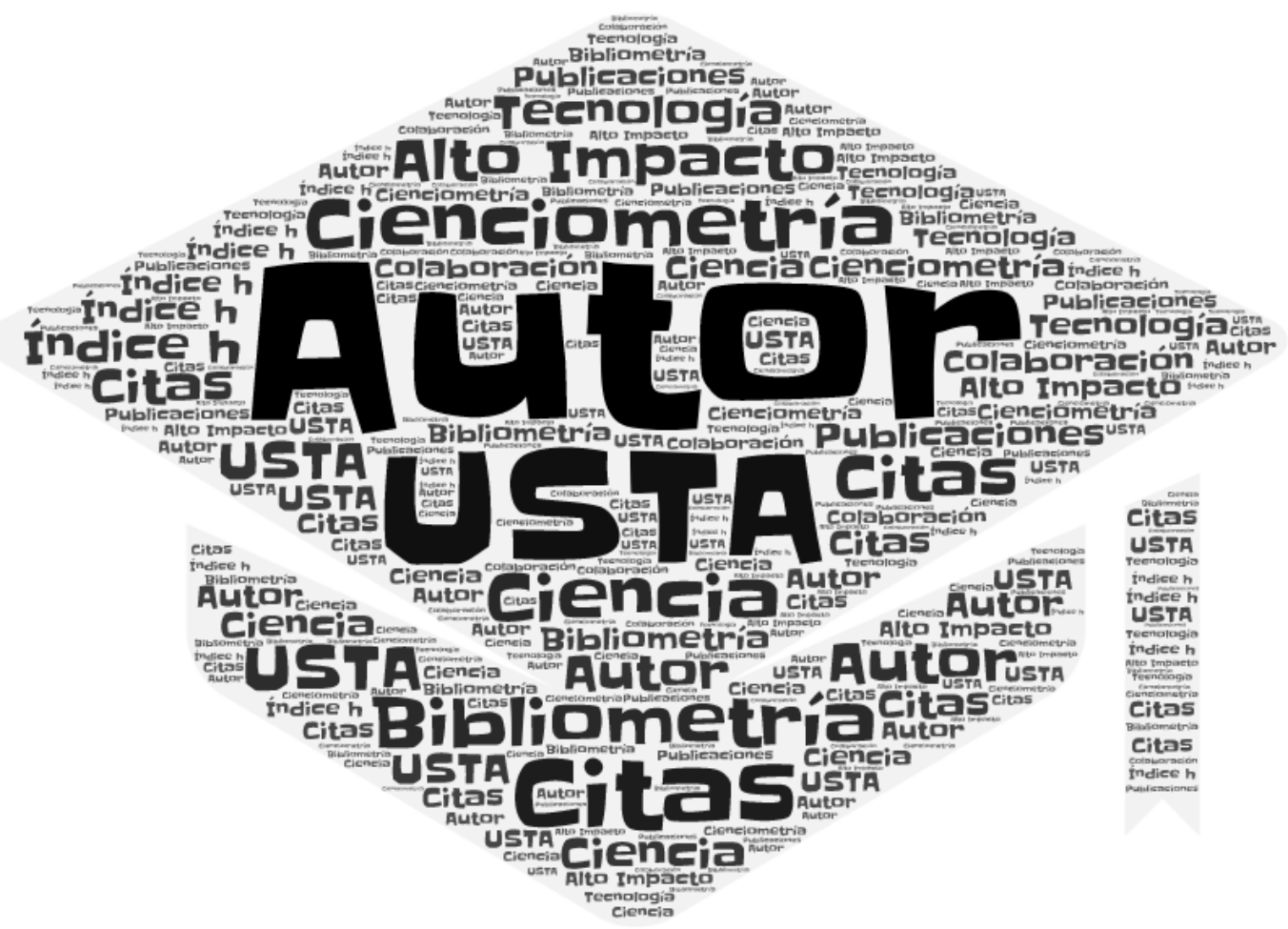

\section{Autor: Vilma Stella Moreno Diaz}

Dirección de Investigación e Innovación - Sede principal Centro de Recursos para el Aprendizaje y la Investigación CRAIUSTA

Por: Cristian Alexander Vega Mosquera y Luz Marina Paez 


\section{Observatorio de bibliometría y cienciometría USTA}

\section{RESUMEN}

Informe de las métricas de autor de la Dra. Vilma Stella Moreno Diaz de las publicaciones indexadas en Google Académico cuyo objetivo es entregar un insumo para el fortalecimiento de las capacidades y potencialidades de los autores de la Universidad Santo Tomás en el posicionamiento y visibilidad de sus publicaciones.

Palabras Claves: Bibliometría, Cienciometría, Autor, Visibilidad académica.

\section{ABSTRACT}

Report of the author metrics of Vilma Stella Moreno Diaz of the publications indexed in Google Scholar whose objective is to provide an input for the strengthening of the capacities and potentialities of the authors of the Santo Tomás University in the positioning and visibility of their publications.

Keywords: Bibliometric, Cienciometric, Journal, Author, Academic visibility. 


\section{Observatorio de bibliometría y cienciometría USTA}

Autor: Vilma Stella Moreno Diaz

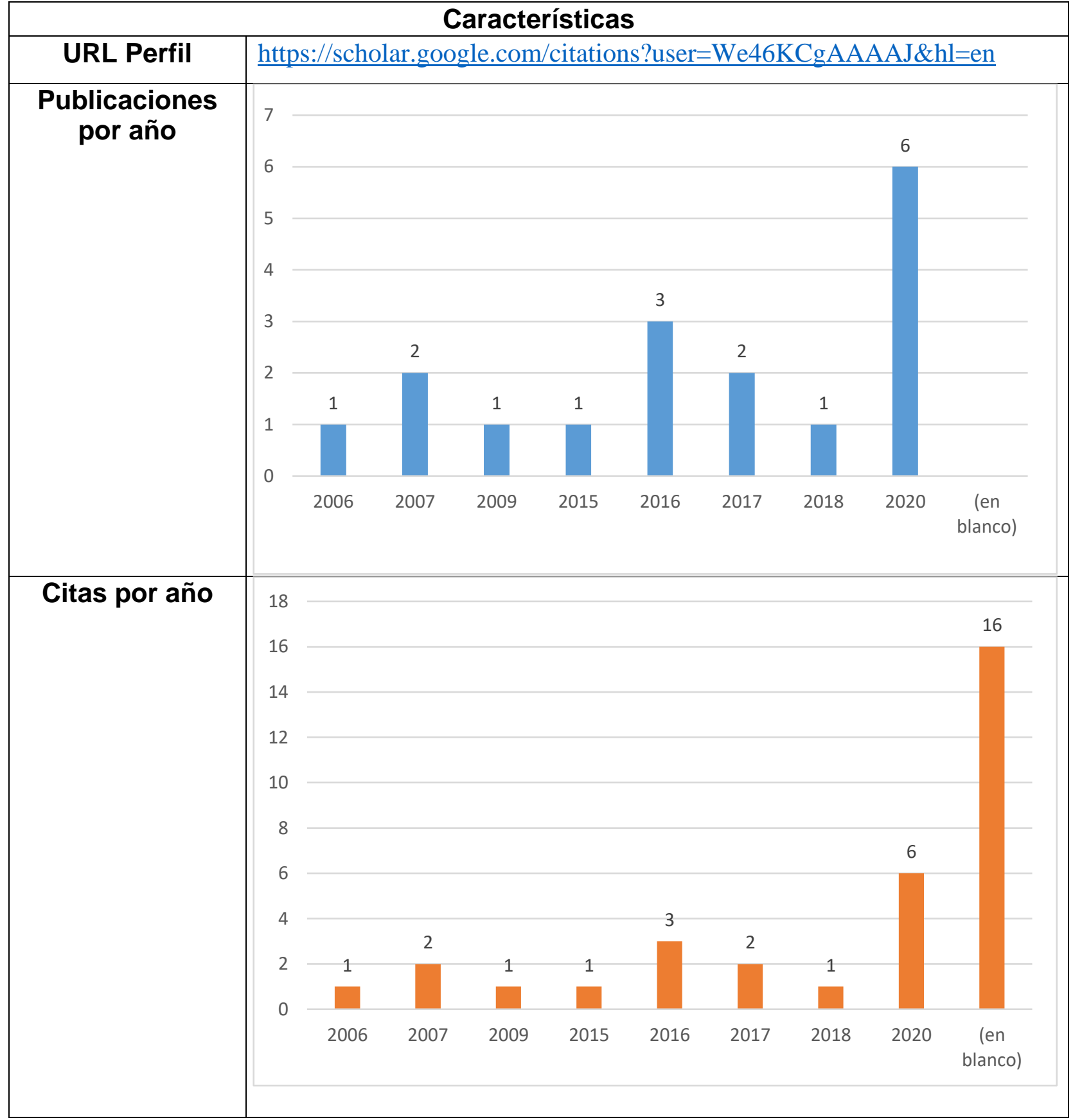




\section{Observatorio de bibliometría y cienciometría USTA}

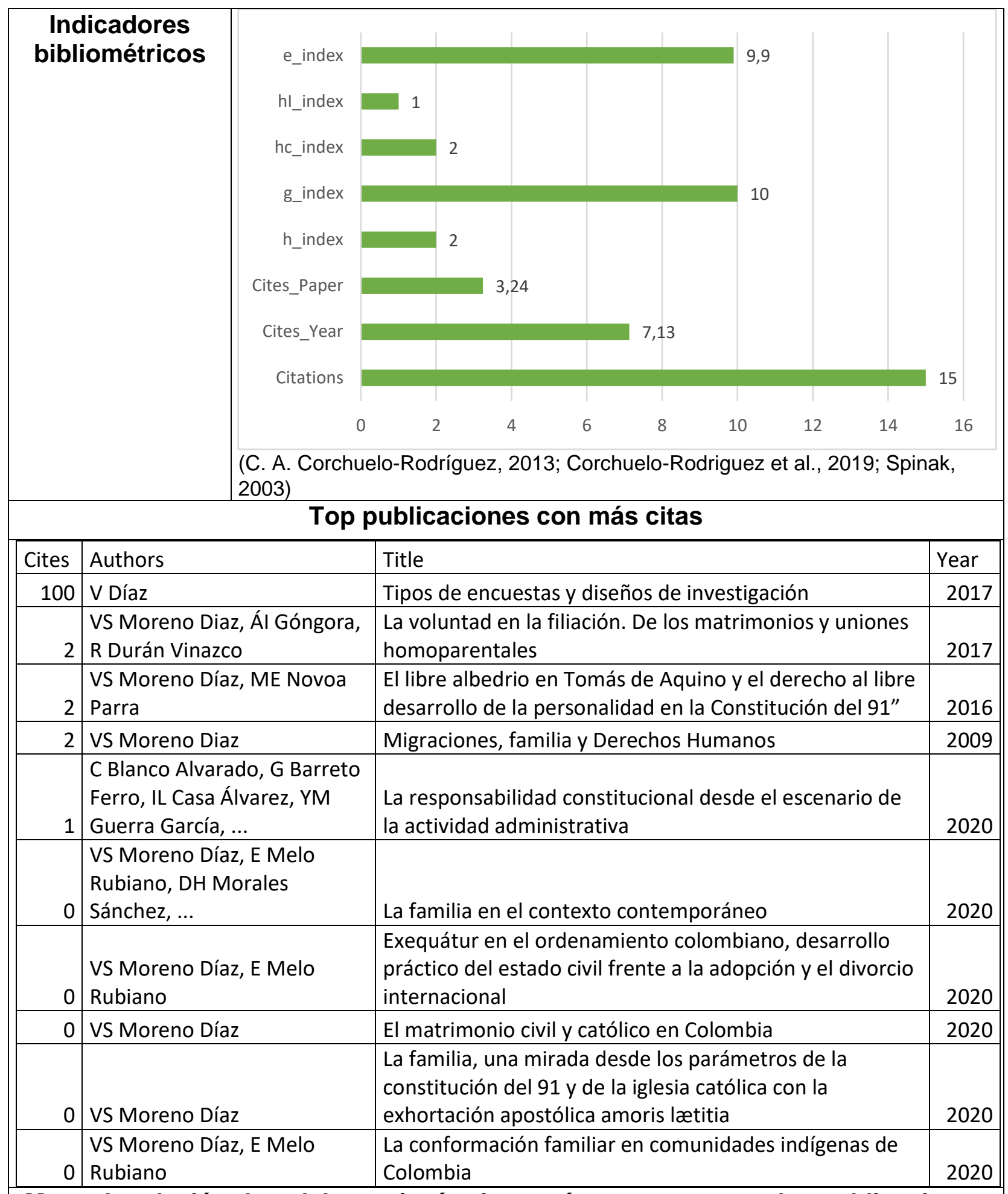

Mapa de relación de palabras y/o términos más recurrentes en las publicaciones 


\section{Observatorio de bibliometría y cienciometría USTA}

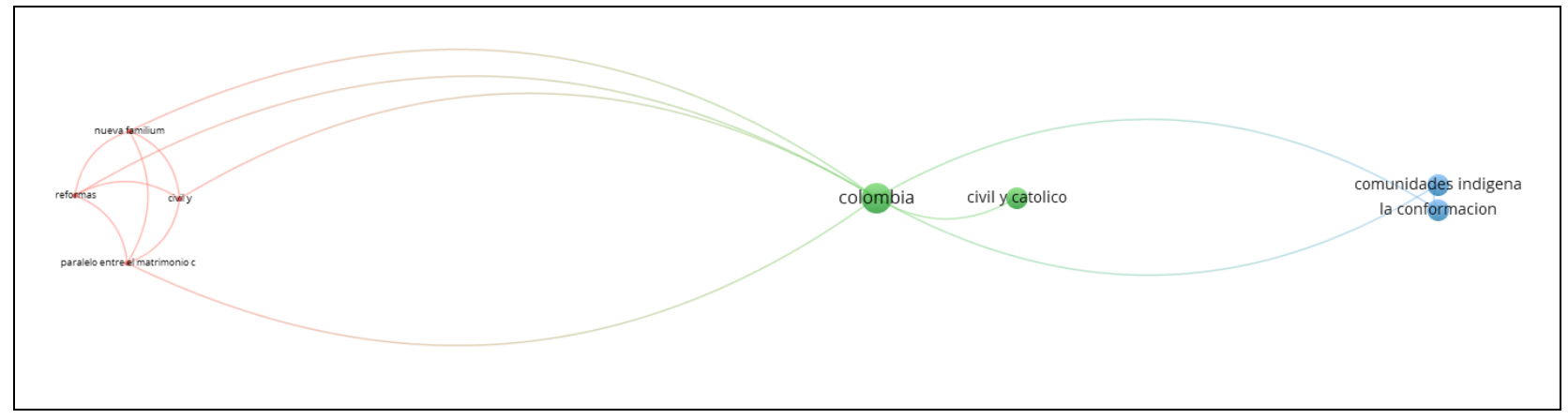

Bibliografía analizada

Alvarado, C. B., Ferro, G. B., Álvarez, I. C., García, Y. G., \& ... (2020). La responsabilidad constitucional desde el escenario de la actividad administrativa. Universidad Santo Tomás, Query date: 2021-02-17 10:17:17.

https://scholar.google.com/citations?view_op=view_citation\&hl=en\&user=We46K CgAAAAJ\&pagesize=100\&citation_for_view=We46KCgAAAAJ:09LM3QYkMK $\mathrm{UC}$

Avila, J. T., Villabona, A. R., Moreno, M. M., \& ... (2018). FODEIN 2018-Informe parcial de investigación.

https://scholar.google.com/citations?view_op=view_citation\&hl=en\&user=We46K CgAAAAJ\&pagesize=100\&citation_for_view=We46KCgAAAAJ:fbc8zXXH2BU $\mathrm{C}$

Camilo, S. L., Nelson, Oscar, P. V., \& Gómez-Jaramillo, A. (2018). Elementos para una justicia de paz restaurativa. Ediciones USTA.

CAPÍTULO, V., EXEQUÁTUR, E., \& ADOPCIÓN, A. L. (s. f.). Vilma Stella Moreno Díaz2 Edilberto Melo Rubiano3. 


\section{Observatorio de bibliometría y cienciometría USTA}

https://scholar.google.com/citations?view_op=view_citation\&hl=en\&user=We46K

CgAAAAJ\&pagesize=100\&citation_for_view=We46KCgAAAAJ:RJujIP1NYNUC

Cuéllar, A. F. (2012). Un mundo sin cárceles es posible, de Alejandro Gómez Jaramillo.

Crítica Penal y Poder, O(2), Article 2.

https://revistes.ub.edu/index.php/CriticaPenalPoder/article/view/2952

Díaz, V. (2017). Tipos de encuestas y diseños de investigación.

https://scholar.google.com/citations?view_op=view_citation\&hl=en\&user=We46K

CgAAAAJ\&pagesize=100\&citation_for_view=We46KCgAAAAJ:T_0gP6tLVL0C

Diaz, V. M. (2006). Mujer: Igualdad entre iguales. Via Inveniendi Et Iudicandi Revista

Virtual, 2(1), 1-1.

Diaz, V. M. (2007). LAS SENTENCIAS DE LA CORTE CONSTITUCIONAL COMO

FUENTE FORMAL DE DERECHO. Nova Et Vetera, Query date: 2021-02-17

10:17:17, 15-40.

Diaz, V. M. (2009). Migraciones, familia y Derechos Humanos. Iusta, 2(31), 123-139.

Diaz, V. M. (2016a). "El Motu proprio del Papa Francisco que derogó la Consulta obligatoria para las Sentencias de Nulidad del Matrimonio Católico. Misión Jurídica, Query date: 2021-02-17 10:17:17, 249-271.

Diaz, V. M. (2016b). Maternidad subrogada perspectiva en el derecho canónico. CRITERIOS. Cuadernos de Ciencias Jurídicas y Política Internacional, 9(9), 51-74.

Diaz, V. M., Góngora, Á., \& Vinazco, R. D. (2017). La voluntad en la filiación. De los matrimonios y uniones homoparentales. Justicia constitucional. Tomo II, Query date: 2021-02-17 10:17:17, 15-38.

Díaz, V Moreno. (s. f.). La institución de la familia en el derecho romano, canónico y civil Métricas de autor 


\section{Observatorio de bibliometría y cienciometría USTA}

colombiano y sus cambios a partir de la constitución de 1991.

https://scholar.google.com/citations?view_op=view_citation\&hl=en\&user=We46K

CgAAAAJ\&pagesize=100\&citation_for_view=We46KCgAAAAJ:ON9TkA72AH

EC

Díaz, VS Moreno. (s. f.). Paralelo entre el matrimonio canónico y el civil y la nueva familia en Colombia. Reformas a la constitución de 1991.

https://scholar.google.com/citations?view_op=view_citation\&hl=en\&user=We46K

CgAAAAJ\&pagesize=100\&citation_for_view=We46KCgAAAAJ:0EnyYjriUFMC

Díaz, VS Moreno. (2007). El matrimonio canónico en su dimensión antropológica.

https://scholar.google.com/citations?view_op=view_citation\&hl=en\&user=We46K CgAAAAJ\&pagesize=100\&citation_for_view=We46KCgAAAAJ:1SLsV1MU4ZU $\mathrm{C}$

Díaz, VS Moreno. (2020a). El matrimonio civil y católico en Colombia. La familia en el contexto contemporáneo, Query date: 2021-02-17 10:17:17.

https://scholar.google.com/citations?view_op=view_citation\&hl=en\&user=We46K CgAAAAJ\&pagesize $=100 \&$ citation_for_view=We46KCgAAAAJ:JTtNqH-x4gYC

Díaz, VS Moreno. (2020b). La familia, una mirada desde los parámetros de la constitución del 91 y de la iglesia católica con la exhortación apostólica amoris lætitia. La familia en el contexto contemporáneo, Query date: 2021-02-17 10:17:17. https://scholar.google.com/citations?view_op=view_citation\&hl=en\&user=We46K CgAAAAJ\&pagesize=100\&citation_for_view=We46KCgAAAAJ:R6EwkKsDylY $\mathrm{C}$

Díaz, VS Moreno, Góngora, Á., Vinazco, R. D., Vargas, L. M., \& ... (s. f.). Justicia Métricas de autor 


\section{Observatorio de bibliometría y cienciometría USTA}

Constitucional Tomo II. Universidad Santo Tomás, Query date: 2021-02-17 10:17:17.

https://scholar.google.com/citations?view_op=view_citation\&hl=en\&user=We46K CgAAAAJ\&pagesize=100\&citation_for_view=We46KCgAAAAJ:CMPXdcK5v8E $\mathrm{C}$

Díaz, VS Moreno, \& Parra, M. N. (2016). El libre albedrio en Tomás de Aquino y el derecho al libre desarrollo de la personalidad en la Constitución del 91 ”. https://scholar.google.com/citations?view_op=view_citation\&hl=en\&user=We46K CgAAAAJ\&pagesize=100\&citation_for_view=We46KCgAAAAJ:IPDSu1ZU3VA $\mathrm{C}$

Díaz, VS Moreno, \& Rubiano, E. M. (2020a). Exequátur en el ordenamiento colombiano, desarrollo práctico del estado civil frente a la adopción y el divorcio internacional. La familia en el contexto contemporáneo, Query date: 2021-02-17 10:17:17. https://scholar.google.com/citations?view_op=view_citation\&hl=en\&user=We46K CgAAAAJ\&pagesize=100\&citation_for_view=We46KCgAAAAJ:aNch6Af-aFkC

Díaz, VS Moreno, \& Rubiano, E. M. (2020b). La conformación familiar en comunidades indígenas de Colombia. La familia en el contexto contemporáneo, Query date: 2021-02-17 10:17:17. https://scholar.google.com/citations?view_op=view_citation\&hl=en\&user=We46K CgAAAAJ\&pagesize=100\&citation_for_view=We46KCgAAAAJ:rJyh6hJnyfgC

Díaz, VS Moreno, Rubiano, E. M., Sánchez, D. M., \& ... (2020). La familia en el contexto contemporáneo.

https://scholar.google.com/citations?view_op=view_citation\&hl=en\&user=We46K Métricas de autor 


\section{Observatorio de bibliometría y cienciometría USTA}

CgAAAAJ\&pagesize=100\&citation_for_view=We46KCgAAAAJ:svGagg1hbZMC

Díaz, VSM. (s. f.-a). EL MATRIMONIO CIVIL Y CATOLICO EN COLOMBIA59. La

familia en el contexto contemporáneo, 95, Query date: 2021-02-17 10:17:17.

https://scholar.google.com/citations?view_op=view_citation\&hl=en\&user=We46K

CgAAAAJ\&pagesize=100\&citation_for_view=We46KCgAAAAJ:J6OZcwVsj5AC

Díaz, VSM. (s. f.-b). LA FAMILIA, UNA MIRADA DESDE LOS PARÁMETROS DE

LA CONSTITUCIÓN DEL 91 Y DE LA IGLESIA CATÓLICA CON LA

EXHORTACIÓN APOSTÓLICA AMORIS LÆTITIA38. La familia en el contexto contemporáneo, 66, Query date: 2021-02-17 10:17:17.

https://scholar.google.com/citations?view_op=view_citation\&hl=en\&user=We46K CgAAAAJ\&pagesize=100\&citation_for_view=We46KCgAAAAJ:vkuYBMKU6w EC

Díaz, VSM, Góngora, Á., \& Vinazco, R. (s. f.). LA VOLUNTAD EN LA FILIACION DE LOS MATRIMONIOS Y UNIONES HOMOPARENTALES1. JUSTICIA CONSTITUCIONAL, 15, Query date: 2021-02-17 10:17:17. https://scholar.google.com/citations?view_op=view_citation\&hl=en\&user=We46K CgAAAAJ\&pagesize $=100 \&$ citation_for_view=We46KCgAAAAJ:v6PuF9mNY3o $\mathrm{C}$

Díaz, VSM, \& Rubiano, E. (s. f.-a). EXEQUÁTUR EN EL ORDENAMIENTO COLOMBIANO, DESARROLLO PRÁCTICO DEL ESTADO CIVIL FRENTE A LA ADOPCIÓN Y EL DIVORCIO INTERNACIONAL112. La familia en el contexto contemporáneo, 129, Query date: 2021-02-17 10:17:17. https://scholar.google.com/citations?view_op=view_citation\&hl=en\&user=We46K Métricas de autor 


\section{Observatorio de bibliometría y cienciometría USTA}

CgAAAAJ\&pagesize=100\&citation_for_view=We46KCgAAAAJ:DwWRdxKAo4C

Díaz, VSM, \& Rubiano, E. (s. f.-b). LA CONFORMACIÓN FAMILIAR EN COMUNIDADES INDÍGENAS DE COLOMBIA1. La familia en el contexto contemporáneo, 29, Query date: 2021-02-17 10:17:17. https://scholar.google.com/citations?view_op=view_citation\&hl=en\&user=We46K CgAAAAJ\&pagesize=100\&citation_for_view=We46KCgAAAAJ:anDooRL1HQE $\mathrm{C}$

Díaz, VSM, \& Sánchez, D. (s. f.). LA CONSOLIDACIÓN DE LA PAZ EMPIEZA EN EL FORTALECIMIENTO DE LA FAMILIAI. https://scholar.google.com/citations?view_op=view_citation\&hl=en\&user=We46K CgAAAAJ\&pagesize=100\&citation_for_view=We46KCgAAAAJ:idthP5jqfYAC

Gómez Jaramillo, A., \& Silva García, G. (2015). Consideraciones metodológicas. Gómez Jaramillo, A. \& Silva García, G. (2015). El futuro de la criminología crítica. Bogotá: Universidad Católica de Colombia. https://repository.ucatolica.edu.co/handle/10983/18177

Gómez Jaramillo, Alejandro, \& Silva García, G. (2015). Críticas y respuestas. Gómez Jaramillo, A. \& Silva García, G. (2015). El futuro de la criminología crítica. Bogotá: Universidad Católica de Colombia. https://repository.ucatolica.edu.co/handle/10983/18176

Gómez-Jaramillo, A. (2018). Populismo, obediencia y divergencia. Utop\&iacute; a y Praxis Latinoamericana, 23(S1), 33-49.

Gómez-Jaramillo, A., \& Montes, R. V. (2019). Crisis de la prisión en Colombia y derechos Métricas de autor 


\section{Observatorio de bibliometría y cienciometría USTA}

humanos. Opción, 35, 663-711.

Gómez-Jaramillo, A., \& Silva García, G. (2015). El carácter rizomático que muestra la idea de dispositivo de cuestión criminal. Gómez Jaramillo, A. \& Silva García, G. (2015). El futuro de la criminología crítica. Bogotá: Universidad Católica de Colombia. https://repository.ucatolica.edu.co/handle/10983/18178

Montes, R Velandia, Alvarez, J. N., León, N. S., \& ... (s. f.). Proyectos de investigaciónDecimotercera convocatoria FODEIN 2018. https://scholar.google.com/citations?view_op=view_citation\&hl=en\&user=We46K CgAAAAJ\&pagesize=100\&citation_for_view=We46KCgAAAAJ:sfsSB71Kuh0C

Montes, Rafael Velandia, Gómez-Jaramillo, A., \& Aristizábal, D. M. B. (2020). El delito de pánico económico en Colombia. Verba luris, 43, 159-174. https://doi.org/10.18041/0121-3474/verbaiuris.43.6507

Moreno, M. B., Navarrete, L. L., Durán, Á. M., \& ... (s. f.). Proyectos de investigaciónDecimotercera convocatoria FODEIN 2019. https://scholar.google.com/citations?view_op=view_citation\&hl=en\&user=We46K CgAAAAJ\&pagesize=100\&citation_for_view=We46KCgAAAAJ:eIKNFFVQvJA $\mathrm{C}$

Novoa, M. P., \& Diaz, V. M. (2015). El libre albedrio en Tomás de Aquino y su relación con el Derecho al Libre desarrollo de la personalidad en la Constitución del 91. Editorial Bonaventuriana, Query date: 2021-02-17 10:17:17. https://scholar.google.com/citations?view_op=view_citation\&hl=en\&user=We46K CgAAAAJ\&pagesize $=100 \&$ citation_for_view=We46KCgAAAAJ:J-pR_7NvFogC

Ordóñez Vásquez, T., Gómez-Jaramillo, A., \& Bayona Aristizábal, D. M. (2019). 


\section{Observatorio de bibliometría y cienciometría USTA}

HALLAZGOS FISCALES Y PROCESOS DE RESPONSABILIDAD FISCAL EN

COLOMBIA 2012-2017. Revista republicana, 27, 211-233.

https://doi.org/10.21017/rev.repub.2019.v27.a74

Sánchez, D. M., \& Díaz, V. M. (s. f.-a). Capítulo 6: Outsiders y dinámicas de reificación en los procesos de consulta previa.

https://scholar.google.com/citations?view_op=view_citation\&hl=en\&user=We46K CgAAAAJ\&pagesize=100\&citation_for_view=We46KCgAAAAJ:Zbx7W2Xs4Qs C

Sánchez, D. M., \& Díaz, V. M. (s. f.-b). Outsiders y dinámicas de reificación en los procesos de consulta previa.

https://scholar.google.com/citations?view_op=view_citation\&hl=en\&user=We46K CgAAAAJ\&pagesize=100\&citation_for_view=We46KCgAAAAJ:xlVdBZVQT58 C

Sánchez, D. M., Díaz, V. M., \& Rubiano, E. M. (s. f.). La familia en el contexto contemporáneo. Universidad Santo Tomás, Query date: 2021-02-17 10:17:17. https://scholar.google.com/citations?view_op=view_citation\&hl=en\&user=We46K CgAAAAJ\&pagesize $=100 \&$ citation_for_view=We46KCgAAAAJ:bkKuixW_xMk C

Torres, A. T., Moreno, M. B., Espitia, L. V., \& ... (s. f.). FODEIN 2017-Informes de investigación.

https://scholar.google.com/citations?view_op=view_citation\&hl=en\&user=We46K CgAAAAJ\&pagesize=100\&citation_for_view=We46KCgAAAAJ:0CzhzZyukY4C

Velandia Montes, R., Gomez Jaramillo, A., Solarte Cucanchón, A., \& Jaramillo Gobanzo, Métricas de autor 


\section{Observatorio de bibliometría y cienciometría USTA}

N. (2018). Los sí delincuentes: Visiones sociales sobre la pena, la criminalidad y el sistema penal. Milla. https://www.ilae.edu.co/web/libros-html/libro-384/index.html

Velandia Montes, Rafael, \& Gómez-Jaramillo, A. (2018). Life imprisonment and predicting behavior. An analysis on crime asainst minors and penal policy in Colombia.

Revista republicana, 25, 241-263. https://doi.org/10.21017/rev.repub.2018.v25.a57 